This is a postprint version of the following published document:

Calle-Silos, F., Valverde-Albacete, F. J., Gallardo-Antolín, A. \& PeláezMoreno, C. (2015). Morphologically filtered power-normalized cochleograms as robust, biologically inspired features for ASR. IEEE/ ACM Transactions on Audio, Speech, and Language Processing, 23(11), 2070-2080.

DOI: http://dx.doi.org/10.1109/TASLP.2015.2464691

(C) 2015 IEEE. Personal use of this material is permitted. Permission from IEEE must be obtained for all other uses, in any current or future media, including reprinting/republishing this material for advertising or promotional purposes, creating new collective works, for resale or redistribution to servers or lists, or reuse of any copyrighted component of this work in other works. 


\title{
Morphologically-filtered power-normalized cochleograms as robust, biologically inspired features for ASR
}

\author{
Fernando de-la-Calle-Silos, Student Member, IEEE, Francisco J. Valverde-Albacete, Member, IEEE, \\ Ascensión Gallardo-Antolín, and Carmen Peláez-Moreno, Member, IEEE
}

\begin{abstract}
In this paper we present advances in the modeling of the masking behavior of the Human Auditory System (HAS) to enhance the robustness of the feature extraction stage in Automatic Speech Recognition (ASR). The solution adopted is based on a non-linear filtering of a spectro-temporal representation applied simultaneously to both frequency and time domains-as if it were an image-using mathematical morphology operations.

A particularly important component of this architecture is the so-called Structuring Element (SE) that in the present contribution is designed as a single three-dimensional pattern using physiological facts, in such a way that closely resembles the masking phenomena taking place in the cochlea. A proper choice of spectro-temporal representation lends validity to the model throughout the whole frequency spectrum and intensity spans assuming the variability of the masking properties of the HAS in these two domains. The best results were achieved with the representation introduced as part of the Power Normalized Cepstral Coefficients (PNCC) together with a spectral subtraction step.
\end{abstract}

This method has been tested on Aurora 2, Wall Street Journal and ISOLET databases including both classical Hidden Markov Model (HMM) and hybrid Artificial Neural Networks (ANN)HMM back-ends. In these, the proposed front-end analysis provides substantial and significant improvements compared to baseline techniques: up to $39.5 \%$ relative improvement compared to MFCC, and $18.7 \%$ compared to PNCC in the Aurora 2 database.

Index Terms-Spectro-temporal processing, Cochlear masking models, Morphological filtering, Automatic speech recognition, Auditory-based features, PNCC.

\section{INTRODUCTION}

$\mathbf{T}$ HE remarkable ability of humans in speech recognition tasks under noisy conditions is still far above that of machines. In this context, several researchers have proposed that modeling the Human Auditory System (HAS) may be an adequate strategy to reduce the gap in performance.

It is well established that feature extraction methods for ASR need to take into account properties of the HAS to a certain extent: the well-known Mel-Frequency Cepstral Coefficients (MFCC) [1], for example, result from non-linear transformations of the frequency domain that mimic Fletcher's psychophysical transfer function [2], and include a triangular

Fernando de-la-Calle-Silos, Ascensión Gallardo-Antolín, and Carmen Peláez-Moreno are with the Department of Signal Theory and Communications, Universidad Carlos III de Madrid, Leganés (Madrid), Spain. (e-mail: fsilos@tsc.uc3m.es, gallardo@tsc.uc3m.es, carmen@tsc.uc3m.es)

F.J. Valverde-Albacete is with the Departamento de Leguajes y Sistemas Informticos, Universidad Nacional de Educación a Distancia, Madrid, Spain filterbank that emulates critical bands in the cochlea. Some other aspects, like the non-linear perception of sound intensity, are also incorporated by means of a logarithmic transformation of the spectrum.

Also widespread, Perceptually-based Linear Prediction (PLP) [3] is a pragmatic approach to model the auditory periphery that includes: resampling for frequency warping, barkscale filter-bank, limited frequency resolution, pre-emphasis according to the threshold of hearing, amplitude compression and smoothing using linear prediction. The computational complexity of PLP feature extraction is similar to MFCC and sometimes provides better recognition accuracy.

There are plenty of other feature extraction methods that take into account the HAS, such as zero crossing peak amplitude (ZCPA) [4], average localized synchrony detection (ALSD) [5], perceptual minimum variance distortionless response (PMVDR) [6], invariant-integration features (IIF) [7], amplitude modulation spectrogram [8], sparse auditory reproducing kernel (SPARK) [9] or the well-known RelAtive SpecTrAl processing (RASTA) [10] that exploits the insensibility of human hearing to slowing varying stimuli by modeling the trend of the auditory periphery to emphasize the transient portions of incoming signals.

On the other hand, a number of detailed physiological models were proposed in the 1980s such as Seneff's auditory model [11] that mimics the nominal auditory-nerve frequency by employing 40 recursive linear filters implemented in cascade and also models the nonlinear transduction from the motion of the basilar membrane to the mean rate of auditorynerve spike discharges, Ghitza's Ensemble Interval Histogram (EIH) model [12] uses the peripheral auditory model proposed by Allen [13] to describe the transformation of sound pressure into the neural rate of firing and focused on the mechanism used to interpret the neural firing rates, or Lyon's model [14], [15] where nonlinear compression, lateral suppression, temporal effects and correlograms are included.

Although these models do not generally provide improved performance on clean speech, they obtain better results than conventional feature extraction methods when speech is degraded, for example, with added noise or reverberation. However, a usually higher computational cost and complexity (with a large number of parameters to be tuned) have prevented a more widespread adoption.

PNCCs [16], [17] have been proposed as an alternative to capture the essentials of the HAS without the complexity of 
full psychoacoustical models. They include the use of a powerlaw non-linearity that replaces the traditional logarithmic nonlinearity used in MFCC coefficients and provides a better fit to the onset portion of the rate-intensity curve developed by the model of [18], a noise-suppression algorithm based on asymmetric filtering that suppresses background excitation and a module that carries out a temporal masking by placing a peak for each frequency channel and suppressing the instantaneous power if it falls below that of the envelope. As explained later in the paper, these features provide dramatic performance improvements over conventional MFCCs and their spectrotemporal representation-or cochleogram-will be the base for our developments.

Other methods also include procedures that emulate HAS masking: in contrast with PNCC that only includes temporal masking, simultaneous or frequency masking is considered in [19] where a frequency-dependent masking threshold is computed, or [20] that performs an estimation of the clean signal taking into account masking effects. In [21] both temporal and simultaneous masking are incorporated performing a timefrequency noise spectral subtraction.

Though most of the algorithms described above include spectro-temporal notions, these are incorporated in separate stages of the processing pipeline. The idea of simultaneously performing temporal and spectral analysis to yield so-called spectro-temporal features has lately emerged, e.g. spectrotemporal Gabor features [22], [23], [24], HIerarchical SpectroTemporal (HIST) [25], spectro-temporal derivative features [26] or sparse spectro-temporal features [27]. Auditoryinspired representations in these domains are reviewed in [28].

Finally, noise robustness techniques are pervasive in ASR, some of them based on the (partial) suppression of background noise from the speech signal in a preprocessing stage. Most of these methods operate on the frequency-domainlike the already mentioned SS [29], Wiener filtering [30] or the minimum mean-square error short-time spectral amplitude estimator [31] —and attempt to enhance the speech signal without extensive modeling of the HAS properties.

As in these previously mentioned works, we also hypothesize that mimicking the Human Auditory System (HAS) may contribute to improve the performance of ASR systems in noisy conditions. Specifically, in this paper we model the masking behavior of the HAS to enhance the robustness of the feature extraction stage in ASR. Despite ingrained intuitions that masking deteriorates signal quality, we propound that it smooths away some noise and artifacts.

The three cornerstones of our procedure are first, the use of mathematical morphology operations to emulate the masking processing of the cochlea, second, the design of $a$ single auditory-inspired three-dimensional mask independent of frequency and intensity and third, the use of an adequate underlying spectro-temporal representation of speech such that the non-linearities in frequency and intensity observed in the auditory masking phenomena are significantly equalized licensing a biologically meaningful application of the two previously mentioned elements.

In particular, our model filters a cochleogram-a spectrotemporal representation of speech-as if it were an image, allowing for the simultaneous processing of both dimensions, time and frequency. The morphological filtering procedure we propose aims to reproduce the masking properties of the HAS. For that purpose, the mask - or in mathematical morphology terminology, the structuring element (SE)-reproduces the spectro-temporal masking behavior as induced from wellknown empirical measurements. Thus, the design of the SE is the crux of our approach.

Note that these empirical measurements were either carried out in the spectral or the temporal domains separately, but we need to extrapolate this to both dimensions. In this paper, we present various structuring element designs that aim at closely resembling the auditory masking phenomena taking place in the cochlea and we also refine our hypothesis that morphological filtering produces a smoothing of the spectrotemporal envelope that better models the masking behavior of the cochlea.

In [32], [33] we presented some evidence of this using Morphological Filtering of speech spectrograms with a roughlyapproximated SE. Such rough modeling already yielded an enhancement of the filtered speech both in terms of objective quality measures and ASR performance. Note that, although some work has been carried out in the field of morphological processing of speech spectrograms using dilation across spectral lines to reduce spectral fluctuations [34], such efforts did not take into account the properties of the HAS.

Finally and for simplicity's sake, we employ a single mask across all frequencies and intensities despite the fact that the masking properties are frequency- and sound intensitydependent [35], relying on the underlying spectro-temporal representation to accommodate these effects. The proper choice of this representation is essential in our feature extraction method. We have selected the one recently proposed in [16], [17] as part of the Power-Normalized Cepstral Coefficients (PNCC) in combination with conventional Spectral Subtraction (SS).

In summary, our contribution in this work is the simultaneous spectro-temporal emulation of the HAS masking phenomena by Morphological Filtering (MF) operations maintaining a low computational cost and complexity with very few tuning parameters. A key aspect is the design of a single bio-inspired three-dimensional SE that is used across the board unlike other spectro-temporal techniques that need larger numbers of different bases as in [22], [23], [24], for example, where a reduced set of temporal, spectral and spectro-temporal filters need to be chosen to make it feasible. For this single SE to remain invariant in frequency and intensity we rely on an underlying spectro-temporal representation that already accounts for that variability. In particular, we have borrowed that of PNCC - even improving the temporal masking there included-while maintaining a low computational complexity with respect to the PNCC baseline.

Regarding our previous works, on the one hand, the highly promising results on the Aurora 2 database noisy continuous digits task presented in [36] are now illustrated with a greater detail and, on the other hand, the performance of the proposed front-end on other different tasks, such as the Wall Street Journal and ISOLET databases, is also shown. The use of both 
conventional Hidden Markov Model (HMM) systems in the first two databases and an Artificial Neural Network/Hidden Markov Model (ANN/HMM) hybrid one on the third database, underlies the remarkable improvements of this feature extraction method across different domains and back-ends.

The rest of this paper is organized as follows: Section II introduces the theory and modeling alternatives for the underlying spectro-temporal representation, Section III explains the theoretical and empirical basis of cochlear masking, Section IV describes our three-dimensional model of this phenomenon introducing the basic terminology of Mathematical Morphology and the design of our biologically inspired SE. Finally Section $\mathrm{V}$ presents the results obtained in various datasets followed by some conclusions and further lines of research in Section VI.

\section{SPECTRO-TEMPORAL REPRESENTATION}

As highlighted before, the underlying spectro-temporal representation - the cochleogram-where the morphological filtering will be applied needs to adopt the necessary frequency scaling and intensity normalization to allow for a single SE to be valid across the full spectrum and intensity range. Since our models have been tested in two different auditorymotivated frequency-scaled cochleograms $S(f, t)$ known as Mel-frequency and Power Normalized based spectro-temporal representations, respectively, next follows a detailed review of the possible alternatives.

It is widely accepted that the cochlea carries out a logarithmic compression of the auditory range whereby higher frequency intervals are represented with less detail than lower frequency ranges. This realization stems from experiments to detect critical bands, the frequency bandwidth around a center frequency whose components affect the sound level and pitch perception of the center frequency.

In this light, the notion of an auditory filter-bank relates three concepts:

- A discretization of a frequency range into $N$ bands.

- A choice of the center of the bands to be related to special frequencies or frequency ranges in the inner ear, which entails the definition of a frequency scale.

- A choice of the bandwidths and shapes of the different filters that takes into consideration the notion of critical bands.

The use of logarithmic frequency scales eases the conceptualization of phenomena like masking, and we will consider several scales of logarithmic frequency: Bark, Mel and the Equivalent Rectangular Bandwidth-induced (ERB) scale.

All of them use methods to calculate the critical bandwidths at different center frequencies and at the same time define scales of equal difference in perception of pitches/levels related to those center frequencies.

1) Critical band and critical-band rate scale: The Bark scale was first defined by [37]:

$$
F_{z}(f)=\frac{26.8}{1+\frac{1960}{f}}-0.53,
$$

where $F_{z}$ is in bark units and $f$ in Hz. The cochlear masking models described in Section III, which are derived from a set of psychoacoustic experiments, are defined in terms of the Bark scale.

2) The MEL scale: The Mel scale [38] is a very well-known logarithmic transformation of the frequency scale:

$$
F_{m}(f)=2595 \log _{10}\left(1+\frac{f}{700}\right),
$$

where $F_{m}$ is in mel units and $f$ in Hz. This frequency transformation is in the core of the most popular ASR feature extraction procedure, the MFCC, where a filterbank of triangular overlapping filters uniformly distributed in the mel scale is usually employed. This is one of our choices for testing our thesis as explained in Section V.

3) ERB and ERB-rate: The ERB was defined in [39], [40] as a more adjusted measurement of the critical band:

$$
B W_{E R B}(f)=6.23 \cdot f^{2}+93.39 \cdot f+28.52(f \text { in } \mathrm{kHz}) .
$$

Based upon these bands a new logarithmic scale may be defined, the ERB-rate [41]

$$
F_{E R B}(f)=11.17 \cdot \log \left|\frac{f+0.312}{f+14.675}\right|+43.0(f \text { in } \mathrm{kHz}),
$$

or the $E R B$ number, [39], [40]:

$$
E R B_{N}(f)=21.4 \log (4.37 f+1) .
$$

Alternatively, a filterbank can also be defined in the time domain by its impulse response, e.g. [42]:

$$
h_{f_{c}}(t)=k t^{n-1} \exp (-2 \pi B t) \cos \left(2 \pi f_{c} t+\phi\right),
$$

where $k$ defines the output gain, $n$ is the order of the filter-in the range 3-5 the filter is a good approximation of the human auditory filter-, $B$ defines the bandwidth, $f_{c}$ is the filter's central frequency and $\phi$ is the phase.

This scaling is at the base of the Gammatone filter-bank used in PNCC, among others, an alternative to the one employed in MFCC that we will compare in our experiments (see Section V). According to [43], the impulse response of the Gammatone function provides an excellent fit to the human auditory filter shapes allowing a better modeling of the masking phenomena. Besides, PNCC [16] incorporate a medium-duration power bias subtraction and a power function nonlinearity to obtain the cochleogram, $S(f, t)$.

Dashed boxes in Figure 1 contain the block diagrams of the two spectro-temporal representations considered in this work: Mel-frequency (left) and Power Normalized (right). The outputs of both submodules are the corresponding cochleograms $S(f, t)$ on which further processing with morphological filters is applied as explained in subsection IV-C. Note that spectral subtraction (shadow block after STFT) is not part of the original mel-frequency and power-normalized representations computations, but it is included here as a basic denoising technique (see subsection IV-C).

\section{Cochlear Masking Empirical Results AND MODELS}

The cochlea is the organ that converts the mechanical vibrations in the middle ear to neural impulses. The basilar 


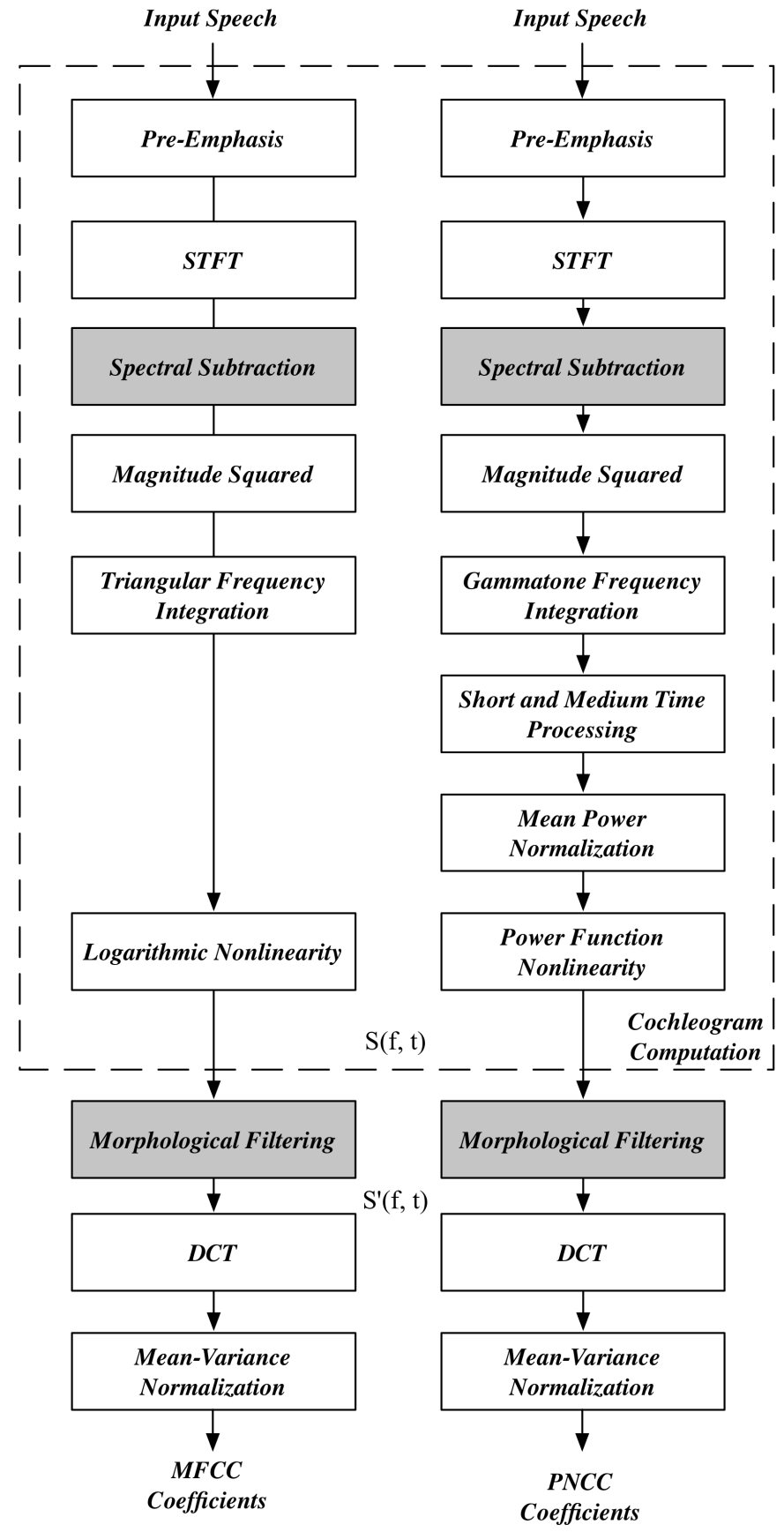

Fig. 1. Structure of the proposed front-end algorithm for the two spectrotemporal representations; the dashed boxes contain the submodules corresponding to the mel-frequency (left) and power-normalized (right) representations; the shaded blocks (Spectral Subtraction (SS) and Morphological Filtering (MF)) indicate the differences regarding conventional MFCC-based and PNCC-based feature extraction.

membrane-the sensing structure that runs the length of the cochlea-has a particular frequency and time response [44].

Cochlear masking is the phenomenon whereby the perception of some frequency at a particular time instant, the masked frequency, is affected by the sound level of another, the masker frequency - possibly at a different time instant_-, to the extent that masked frequencies may disappear from perception.
A masking tone will be defined as

$$
s(F, t)=L_{m} \delta\left(F-F_{m}, t-T_{m}\right),
$$

where $F$ is in any of the transformed frequency scales introduced in the previous section, $L_{m}$ is the sound pressure level of the tone, $F_{m}$ and $T_{m}$ are the masker frequency and time instant and $\delta$ represents the Dirac delta function.

Cochlear masking has been studied as the effect of a masker on simultaneously masked frequencies, simultaneous masking, and as the phenomenon whereby a masker affects non-simultaneous frequencies, temporal masking. Classical masking experiments concentrated in determining the amount of masking in either of these directions-frequency or timein isolation. But it is important to notice that a given (masked) frequency is always being masked by maskers at different time instants - both from earlier and later maskers-and frequencies-both from lower and higher frequency maskers.

\section{A. Simultaneous masking}

Simultaneous masking is defined as the minimum sound pressure level of a test sound, probe or signal-normally a pure tone-that is audible in the presence of a masker. By varying the frequency of the probe throughout the spectrum, a masking pattern may be obtained. An experimental fact is that the shape and sound pressure level $L_{m}$ of the masker is quite determinant of the masking pattern. Regarding the change of masking with masker parameters, [45] noticed that simultaneous masking is better represented in logarithmic scales where the spacing and the masker frequency slopes extend more regularly to either side of the spectrum.

A simultaneous masking model can be extracted from Fig. 6.14 of [35] by fitting slopes for $L_{m}=60 \mathrm{~dB}$ in the Bark scale. We assume a constant $L_{m}$ across all frequencies and intensities, relying on the underlying spectro-temporal representation to accommodate the frequency-intensity dependency of the masking properties.

\section{B. Temporal masking}

Temporal masking has methodologically been treated as two separate processes: premasking occurs before the appearance of the masker while postmasking manifests itself after the masker is no longer present. It is well agreed-upon that premasking is noticeable about $20 \mathrm{~ms}$ prior to the masker, while the duration of postmasking extends well beyond $200 \mathrm{~ms}$, perhaps as far as $500 \mathrm{~ms}$ [32].

Thus, premasking can be modeled as a constant slope of $+25 \mathrm{~dB} / \mathrm{ms}$, starting $20 \mathrm{~ms}$ before the masker. Postmasking can be modeled with the fitted model for single masker-induced postmasking presented in [46],

$$
M\left(t-T_{m}, L_{m}\right)=a\left(b-\log \left(t-T_{m}\right)\right)\left(L_{m}-c\right)
$$

where $M$ is the amount of masking, $t$ is measured in ms, $L_{m}$ is the masker level in $\mathrm{dB} \mathrm{SPL}$, and $a, b$ and $c$ are parameters obtained by fitting the curve to the data. In particular,

- $a$ is related to the slope of the time course of masking.

- $b$ is the logarithmic of the probe-masker delay intercept.

- $c$ is the intercept when masker level is expressed in dBL. 


\section{Smoothed masking responses}

As suggested by the previous sections, an idealized masking model for a masker at $\left(F_{m}, T_{m}\right)$ could be a cone with the appropriate decays in the (logarithmically-)scaled frequency and time coordinates. But findings consistently suggest a masking model that is smooth around $\left(F_{m}, T_{m}\right)$, with sublinear decays close to this point and superlinear decays further away [35]: a sort of apex-smoothed cone.

At this point, it is worth mentioning that it seems that the masking capabilities of the cochlea co-evolved in the presence of a noise that has the peculiarity of raising masking thresholds uniformly, that is, giving a flat frequency response [35].

We hypothesize that at the level of granularity at which the cochlear response is being observed this phenomenon is also present, and the masking response of a particular tone $\left(F_{m}, T_{m}\right)$ must be the non-linear aggregation of many masking responses of other neighboring masking tones $\left(F_{m}+\Delta F, T_{m}+\Delta T\right)$ with $\Delta T<<T_{m}, \Delta F<<F_{m}$ which account for the smooth sublinear decay. This would manifest as a smoothness constraint for models of the masking response in the neighborhood of $\left(F_{m}, T_{m}\right)$. This will be used in Section IV-B to constraint the SE.

\section{A THREE-DIMENSIONAL MODEL OF COCHLEAR MASKING}

\section{A. An overview of morphological processing}

Mathematical Morphology is a theory for the analysis of spatial structures [47] whose main application domain is in Image Processing as a tool for thinning, pruning, structure enhancement, object marking, segmentation and noise filtering [48]. It may be used on both binary and grey-scale images.

To perform MF operations, we first convolve the image with a structuring element and then select the output value depending on the thresholded result of the convolution. In this paper, we apply MF on cochleograms, our underlying spectrotemporal representation, that will be processed as if they were images. This spectro-temporal representation is explained on Section II.

With the proper choice of SE, morphological operations on the cochleogram reproduce the phenomenon of auditory masking where the most prominent or salient elements of the cochleogram mask their surroundings in both the temporal and frequency domain.

Erosion and dilation are the basic morphological operations. Erosion is used to reduce objects, while dilation produces an enlargement and fills in small holes. Let $S$ be the underlying spectro-temporal representation and $M$ the three-dimensional structuring element, erosion is defined as: $S \ominus M$ and dilation: $S \oplus M$.

Erosion and dilation with a general structuring element require relatively simple algorithms and there are fast implementations that allow us to perform such operations efficiently. For gray-scale images, erosion is the minimum over the structuring element and dilation the maximum, respectively. For a pixel at $(n, k)$ where $n$ is the frequency bin and $k$ the time step these operations can be defined as follows:

$$
\begin{aligned}
& (S \ominus M)(n, k)=\min _{(\phi, \tau) \in \mathbb{R}^{2}}\{S(n, k)-M(n-\phi, k-\tau)\} \\
& (S \oplus M)(n, k)=\max _{(\phi, \tau) \in \mathbb{R}^{2}}\{S(n, k)+M(n-\phi, k-\tau)\},
\end{aligned}
$$

where $(\phi, \tau)$ ranges over the domain of definition of $M$.

There are two possible operators generated by the combination of erosion and dilation using the same structuring element for both operations: opening $(S \circ M)$ and closing $(S \bullet M)$. The first one is an erosion followed by a dilation and the second, a dilation followed by an erosion. Mathematically it can be expressed as:

$$
\begin{aligned}
& S \circ M=(S \ominus M) \oplus M, \\
& S \bullet M=(S \oplus M) \ominus M .
\end{aligned}
$$

The opening operator tends to remove the outer tiny leaks and round shapes, whereas the closing operator preserves the regions that have a similar shape as the structuring element. Previous experiments [32] show that closing performs better for ASR than opening.

For producing the final masked cochleogram $S^{\prime}$, first the closing operator is applied on the original (possibly denoised) spectro-temporal representation $S$ using the structuring element $M$ and the result is subsequently added on $S$ :

$$
S^{\prime}=\lambda S+(1-\lambda) S \bullet M .
$$

where $\lambda$ is a configuration parameter that weights both contributions and that has been set to 0.5 in our experiments $(\lambda=1$ indicates no morphological filtering and corresponds with our baseline system). From this enhanced cochleogram $S^{\prime}$, mel-frequency or power-normalized based coefficients are computed following the procedure explained in subsection IV-C and represented in Figure 1.

\section{B. Structuring element}

In this section we describe auditorily motivated structuring elements that try to emulate the complex phenomenon of cochlear masking when used in combination with MF. The $\mathrm{SE}$ acts as the cochlea's response to tone maskers, and the morphological filtering mechanism reproduces the masking itself. Three different structuring elements are presented, the piecewise-linear, piecewise-paraboloid and piecewise-convex models.

The basic piecewise-linear model for masking can be observed in Figure 2.(a) (continuous blue line). This SE is built with linear slopes for the simultaneous masking model and the logarithmic model of Equation 8 for the temporal masking. In this model, referred to as the idealized model of masking in Section III-C, the SE for a single frequency-time point at $(n, k)$ is not smooth.

To be consistent with the smoothness constraint we created two new SE based in 3D quadrics, built by aggregating 4 asymmetric quadric quadrants of different parameters centered at $(n, k)$ fitted to the empirical models in Sections III-A and III-B.

The piecewise-paraboloid model is built by aggregating paraboloid quadrants and the piecewise-convex model using 
(a) Simultaneous masking

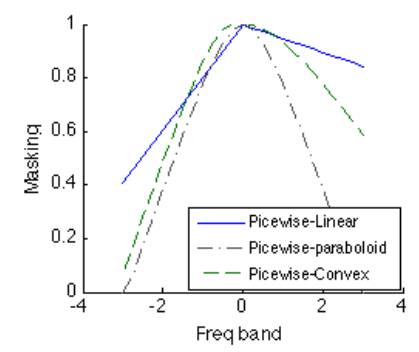

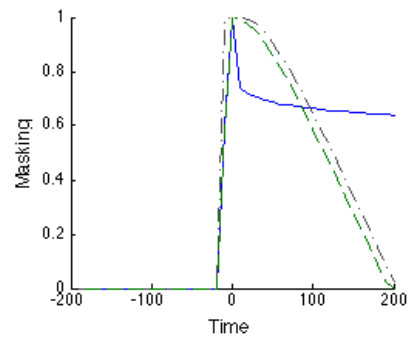

(b) Temporal masking
Fig. 2. Comparison between the piecewise-linear, piecewise-paraboloid and piecewise-convex models in both frequency (left) and time (right) axes.
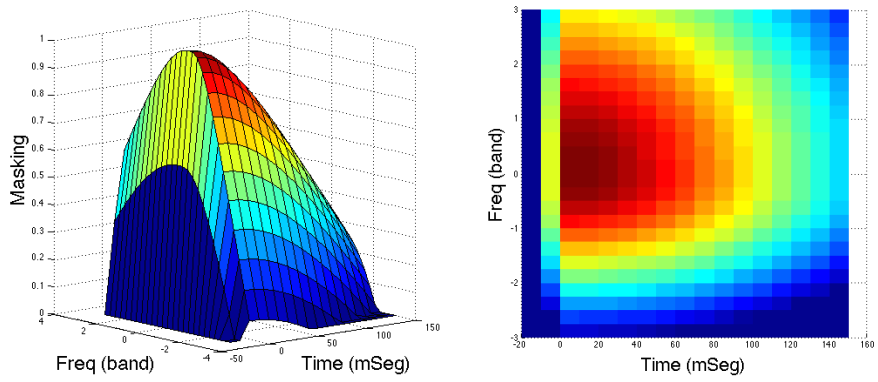

Fig. 3. Three-dimensional representation of the piece-wise convex SE. Color represents the weight of each pixel in the morphological operations. Note how temporal and simultaneous masking are interpolated by the quadrics over the parameters suggested by the pure temporal and frequency models mentioned in in Section III. The asymmetry in the slopes towards higher and lower frequencies - already used in [32] —reflects the choice of different parameters to define the hyperboloids in each quadrant. This effect is more evident in the post-masking than in the pre-masking part of the SE's skirt.

hyperboloid quadrants. A comparison of the masking response of these models with the piecewise-linear model projected onto the time and frequency coordinates can be observed in Figure 2.

As confirmed by the results in Section V, filtering with the piecewise-convex obtains the best performance. Different sizes in both frequency and time scale were tested, and the best performance was obtained by taking $10 \mathrm{~ms}$ of premasking, $150 \mathrm{~ms}$ of postmasking, and 6 bands (in Bark scale) in frequency. The 3D shape of this structuring element can be seen in Figure 3.

Since the cochlear masking model is defined in terms of the Bark scale but the spectro-temporal representations considered in this work are related to the Mel (MFCC) or ERB (PNCC) scales, the appropriate transformations between scales are applied before the morphological processing. Finally, a normalization between zero and one was applied on the intensity dimension and the SE was padded with zeros in the negative time region to center it in the mask around the pixel in which the morphological closing operation is to be performed.

The SE finally chosen can be seen at the upper left of Figure 4(a) at scale, along with examples of the output of some of the processing steps leading to the final cochleogram.

\section{Morphological filtering-based front-ends}

In this subsection, we describe how the morphological filtering is embedded in the whole feature extraction process

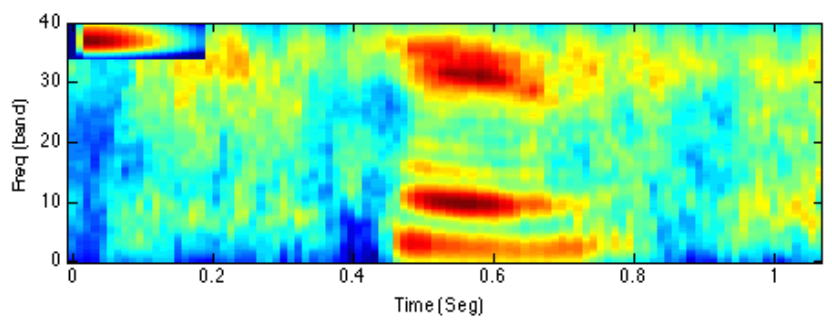

(a) Noisy Spectrogram $S$ compared with the SE (upper left).

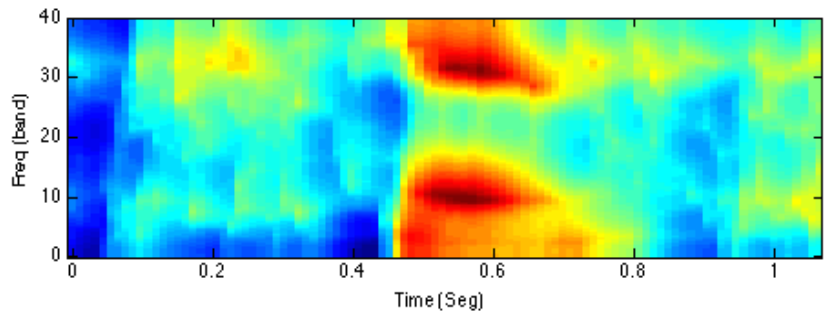

(b) Spectrogram after morphological filtering, $S \bullet M$.

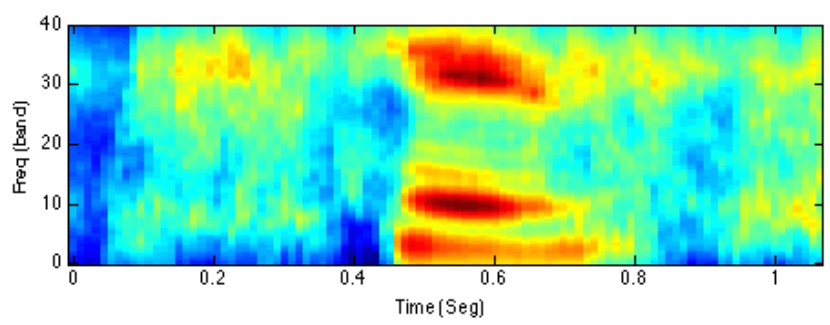

(c) Final cochleogram $S^{\prime}$ with $\lambda=0.5$.

Fig. 4. Choice spectrograms output by each step of the architecture.

for automatic speech recognition.

Figure 1 represents the block diagram of the two complete proposed front-ends based on Mel-frequency (left) and Power Normalized (right) spectro-temporal representations where the shadow blocks are our additions to, respectively, conventional MFCC and PNCC feature extraction: MF and SS. What we call a masked cochleogram, $S^{\prime}(f, t)$, is obtained by performing morphological filtering on $S(f, t)$ using one of the single structuring elements described in subsection IV-B. As for the spectral subtraction block, we found synergies with MF under the MFCC framework in our previous work [32], [33], [49] that we also confirm in this paper for PNCC (see Section V). The last two blocks in both schemes carry out the usual procedure, to de-correlate the resulting filter-bank energies by means of the Discrete Cosine Transform (DCT), followed by a Mean and Variance Normalization (MVN).

\section{EXPERIMENTAL RESULTS}

In this section we present the experiments carried out on three different datasets: Aurora 2, ISOLET and a noisy contaminated version of Wall Street Journal.

\section{A. Feature extraction}

As mentioned before, two different spectro-temporal representations were considered: mel-frequency and powernormalized cochleograms (see Section II). For either type, 


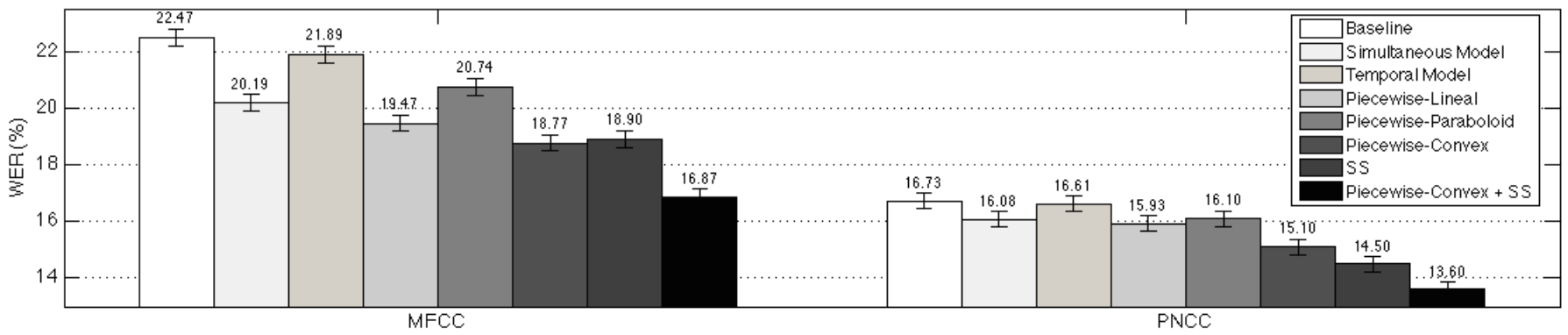

Fig. 5. Recognition results in terms of WER[\%] and 95\% confidence intervals using the AURORA 2 dataset (averaged over all sets).

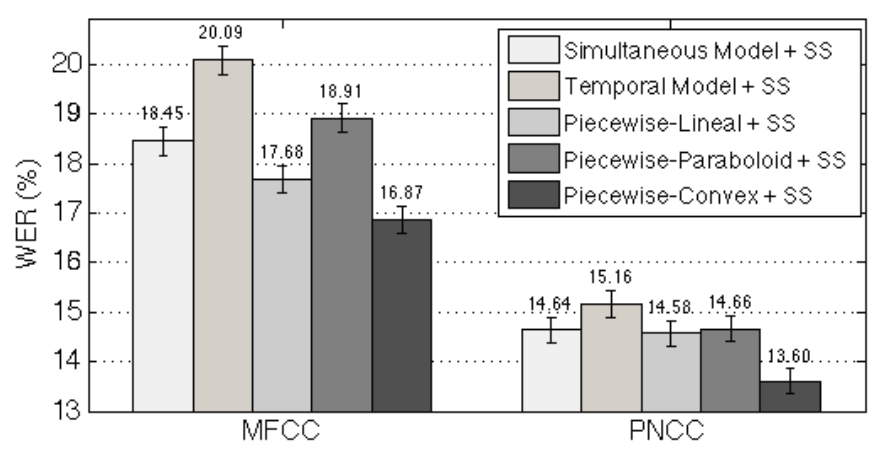

Fig. 6. Recognition results in terms of WER [\%] and 95\% confidence intervals using the AURORA 2 dataset (average over all the sets) for the different structuring elements in combination with spectral subtraction (SS).

speech was analysed using a frame length of $25 \mathrm{~ms}$ and a frame shift of $10 \mathrm{~ms}$. After preemphasis and Hamming windowing an auditory filter bank analysis was applied over the spectrogram computed by using the Short-Time Fourier Transform (STFT). In particular, in the case of the mel-frequency representation, a set of triangular mel-scaled filters were used, whereas, for power-normalized cochleograms a bank of 40 gammatoneshaped filters whose center frequencies are linearly spaced in the ERB scale between $200 \mathrm{~Hz}$ and $4000 \mathrm{~Hz}$ was applied, followed by the PNCC [16] medium-duration power bias subtraction and power function nonlinearity. In both cases, in order to decorrelate the filterbank log-energies obtained in the previous stage, a DCT was computed over them. Cepstral coefficients $C_{0}$ to $C_{12}$ were retained together with their corresponding delta $(\Delta)$ and acceleration $(\Delta \Delta)$ coefficients to yield feature vectors of 39 components. Mean and variance normalizations were applied on each of the components.

When indicated, a conventional SS was employed over the noisy signal in order to emphasise speech over noise and MF applied over the corresponding enhanced cochleograms. Samples of the features files for the different datasets, and the scripts to replicate the results on the Aurora 2 dataset are available at [50].

\section{B. AURORA 2 dataset}

We used the AURORA 2 dataset [51], to test our model, and to select the best structuring element. In particular, the proposed front-ends were tested in mismatched conditions.
AURORA 2 consists of a set of connected digits spoken by American English speakers and recorded at a sample rate of $8 \mathrm{KHz}$. The database was contaminated with a selection of 8 different real-world noises (subway, babble, car, exhibition hall, restaurant, street, airport and train station) at different Signal-to-Noise Ratios (SNR). In particular, SNRs from $0 \mathrm{~dB}$ to $20 \mathrm{~dB}$ with $5 \mathrm{~dB}$ step were considered for our experimentation. The recognizer was based on HTK (Hidden Markov Model Toolkit) software package [52] with the configuration included in the standard experimental protocol of the database described in [51], where a standard Gaussian Mixture Model (GMM)-HMM system with a 16-state word-based HMM and a 5-state silence model was adopted. As our system was tested in mismatched conditions, acoustic models were obtained from the clean training set of the database, whereas test files correspond to the complete test sets A, B and C.

Recognition results in terms of Word Error Rate (WER) and their 95\% confidence intervals are shown in Figure 5. These results correspond to several experiments carried out to study the impact of MF with the SE described in Section IV applied in isolation or in combination with SS and employing mel-frequency or power-normalized based spectro-temporal representations (labeled respectively, as $M F C C$ and $P N C C$ ).

We consider first the influence of MF in ASR system performance with different SEs. From Figure 5, applying MF only in the frequency domain to simulate simultaneous masking (results labeled as Simultaneous Model) produces better results than applying MF only in the temporal domain (results labeled as Temporal Model). The comparison between the three three-dimensional SE considered (piecewise-linear, piecewise-paraboloid and piecewise-convex) indicates that the last one outperforms the other 3D models as well as the baseline and the simultaneous and the temporal models for both spectro-temporal representations and therefore was chosen for the subsequent experiments. In particular, the application of MF with the piecewise-convex SE over noisy spectrograms produces relative error reductions of $16.5 \%$ for $M F C C$ and 9.7\% for PNCC with respect to the corresponding baselines, both statistically significant. This suggests that the proposed model is suitable for representing the robust behavior of the HAS in the presence of noise.

Furthermore, Figure 6 presents the results obtained employing the different proposed SE in combination with SS: the piecewise-convex SE obtained the best performace using 

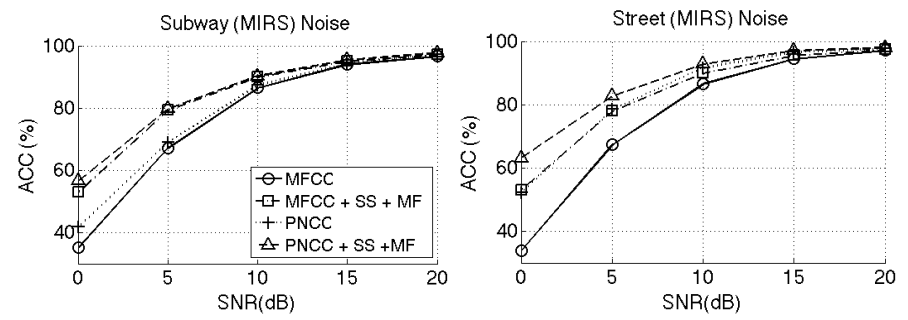

Fig. 8. Recognition results obtained under different convolutive noise conditions in terms of ACC[\%] using the AURORA 2 dataset.

either MFCC or PNCC. For these reasons, from now on in this paper, MF will refer to morphology filtering with the piecewise-convex $\mathrm{SE}$.

Secondly, we also investigated combinations of SS and MF. As expected, for both spectro-temporal representations, SS with no MF clearly outperforms the corresponding baselines. For both, MFCC and PNCC, the joint use of SS and MF improves the recognition rates obtained with $\mathrm{SS}$ in a statistically significant manner. In particular, for $M F C C$ the relative error reduction achieved by $\mathrm{MF}+\mathrm{SS}$ with respect to $\mathrm{SS}$ is $10.7 \%$ and $24.9 \%$ with respect to the baseline. The relative error reduction obtained with $P N C C$ is $6.2 \%$ and $18.7 \%$ related to using only SS and the baseline, respectively. These results show that a positive synergy exists between the SS and MF techniques. Other spectral suppression methods like MMSE [31] and Wiener [30] filtering were also initially tested but yielded worse results than SS in conjunction with PNCC.

Third, the comparison of both spectro-temporal representations shows that the different versions of features based on PNCC (baseline, SS, MF, SS+MF) achieve in all cases better recognition rates than the corresponding features based on $M F C C$. The best combination of PNCC (MF+SS) produces a relative error reduction of $19.4 \%$ with respect to the best combination of $\mathrm{MFCC}(\mathrm{MF}+\mathrm{SS})$ and of $39.5 \%$ with respect to the $M F C C$ baseline. Also, it is worth noting that even PNCC in isolation obtains similar results than the best combination of $M F C C$-based features $(\mathrm{MF}+\mathrm{SS})$.

Figure 7 and Figure 8 show the recognition Accuracy (ACC) for each type of noise and SNR. For brevity's sake, only the results obtained by the baselines and $\mathrm{MF}+\mathrm{SS}$ are shown in these figures. It can be observed that the PNCC $(\mathrm{MF}+\mathrm{SS})$ method achieves the best performance in almost every noise and SNR conditions. In some cases the MFCC method $(\mathrm{MF}+\mathrm{SS})$ achieves similar results to $P N C C$ as can be gleaned from Figures 7 (d), (f), (h) and Figure 8 (b). Results in the presence of convolutional noise as in Figure 8 show no degradation compared to the results obtained in the presence of additive noise only.

To conclude, we have achieved a better relative error reduction in the AURORA 2 database than some other stateof-the-art techniques; for instance, 2D-Gabor features based on power-normalized spectrograms achieve a relative error reduction of only $7.04 \%$ compared to PNCC using a HMM back-end [23].

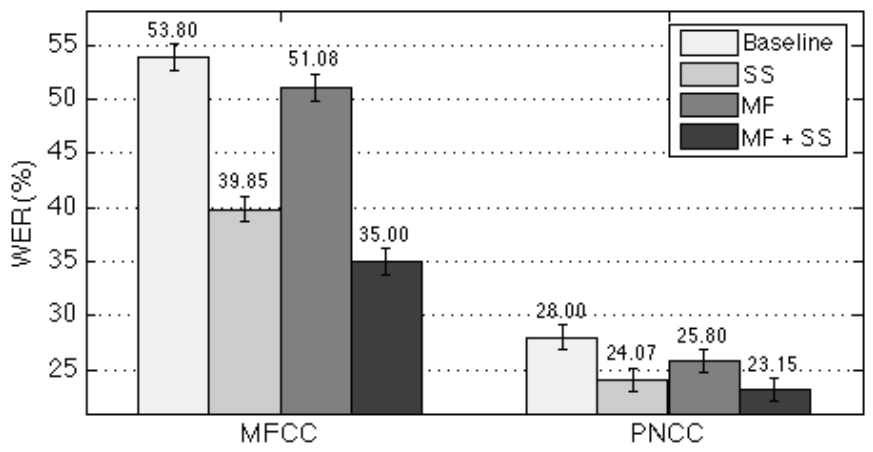

Fig. 9. Recognition results in terms of WER [\%] and 95\% confidence intervals using the ISOLET dataset (average over all the noises and SNR, tested in mismatched conditions).

\section{ISOLET dataset}

In this section, we present the experiments carried out on the ISOLET database [53]. This database consists of 7800 English alphabet spoken letters (two productions of each letter per each of the 150 speakers) at a sample rate of $16 \mathrm{KHz}$. Specifically, we used a version of this database called noisyISOLET [54] where the original ISOLET was contaminated with 8 different noise types from the NOISEX database at several SNRs (clean, 0, 5, 10, 15 and $20 \mathrm{~dB}$ ). The noise types are: speech babble, factory noises 1 and 2, car, pink, F-16 cockpit, destroyer operations room and military vehicle noise.

The experiments were performed using the ISOLET testbed described in [54]. In particular, we trained a hybrid MultiLayer Perceptron (MLP)-HMM system [55] using a context of 5 frames to yield an MLP input dimension of 195 and only one hidden layer is employed. We employed the Quicknet multilayer perceptron (MLP) package for acoustic modeling [56].

This system was tested in mismatched conditions where the system is trained using clean speech and the test set consists of speech contaminated with a balanced combination of the previously mentioned noises at several SNRs. A 5-fold leave-one-out procedure was used to improve the statistical significance of the results. The corresponding recognition results in terms of WER and their 95\% confidence intervals are shown in Figure 9.

We obtained similar results to those using the AURORA 2 dataset, as can be seen in Figure 9 where, first, SS alone (without MF) clearly outperforms the corresponding baselines for both types of spectro-temporal representation (MFCC and $P N C C$-based). Second, the application of MF increases the recognition rates with respect to the corresponding baselines for both representations. Third, the joint use of SS and MF improves the recognition rates obtained with SS in a statistically significant manner. And last, the PNCC features (baseline, SS, $\mathrm{MF}, \mathrm{SS}+\mathrm{MF}$ ) achieve in all cases better recognition rates than the corresponding features based on $M F C C$.

With this set of experiments we have shown that the proposed front-ends achieve also good results in hybrid ASR systems. Besides, in comparison with our previous work over the ISOLET database [32], it can be observed that we have successfully improved the design of the three-dimensional SE by means of the incorporation of perceptual facts, yielding better results. 


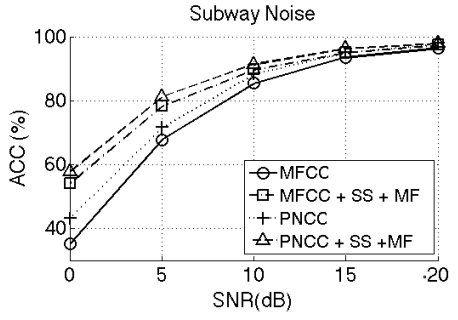

(a)

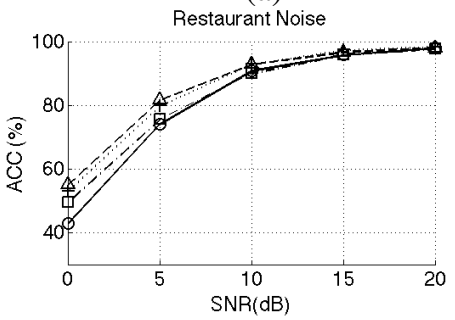

(e)

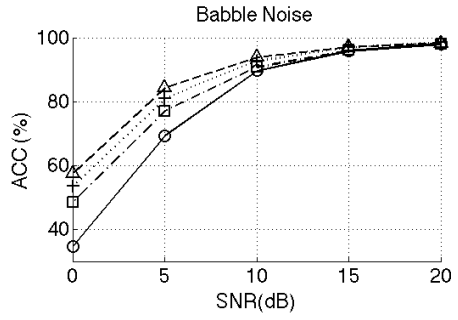

(b)

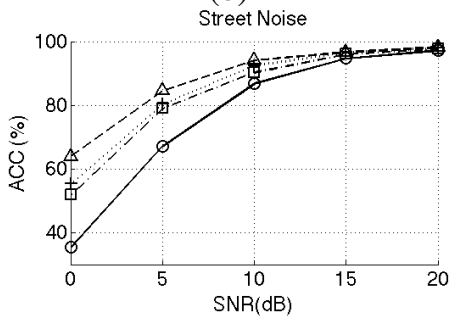

(f)

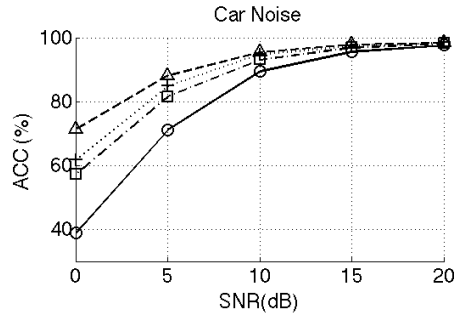

(c)

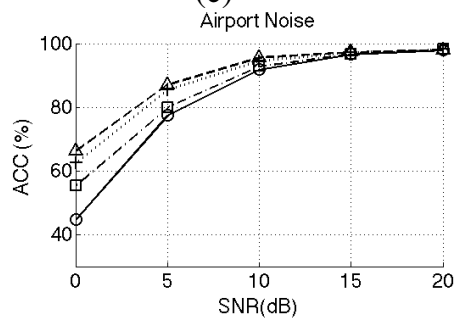

(g)

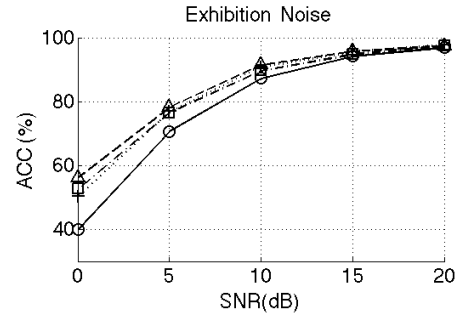

(d)

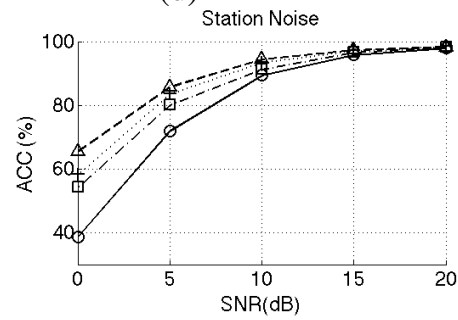

(h)

Fig. 7. Recognition results obtained under different additive noise conditions in terms of ACC[\%] using the AURORA 2 dataset.

\section{WSJO dataset}

In this section, we present the experiments carried out on the Wall Street Journal (WSJ) database, consisting of read speech from a machine-readable corpus of WSJ news text [57]. The experiments were performed using the Hidden Markov Model Toolkit (HTK) recipe described in [58], employing a tri-gram language model with $5 \mathrm{k}$ vocabulary size and the Carnegie Mellon University (CMU) pronunciation dictionary.

To test the robustness of the different methods we used the same four standard testing environments as [17]: (1) white noise, (2) noise recorded live on urban streets, (3) single-speaker interference and (4) background music. The street noise was recorded on streets with steady but moderate traffic. The masking signal used for single-speaker-interference experiments consisted of other utterances drawn from the same database as the target speech, and background music was selected from music segments from the original Defense Advanced Research Projects Agency (DARPA) Hub 4 Broadcast News database.

For training the acoustic models, we used the WSJ0 SI84 training set which contains 7308 clean recordings (14 h). The different front-ends were tested on noisy versions of the WSJ0 $5 \mathrm{~K}$ test set, obtained by digitally adding the previously mentioned noises-white, street, speaker and music - to the corresponding clean speech at four different SNRs using the FaNT tool [59] (with G.712 filtering). All the noise tests are evaluated in mismatched conditions (that is, training on clean speech and testing on noisy speech).

Recognition results in terms of WER and their 95\% confidence intervals are shown in Figure 10. These results correspond to the average over all the noises and SNR conditions outlined above. The performances of our systems on clean speech employing the WSJ0 5K test set are: 5.36\% WER for MFCC and $6.67 \%$ WER for PNCC.

Figure 11 shows the recognition Accuracy (ACC) for each type of noise and SNR. For brevity's sake only the results

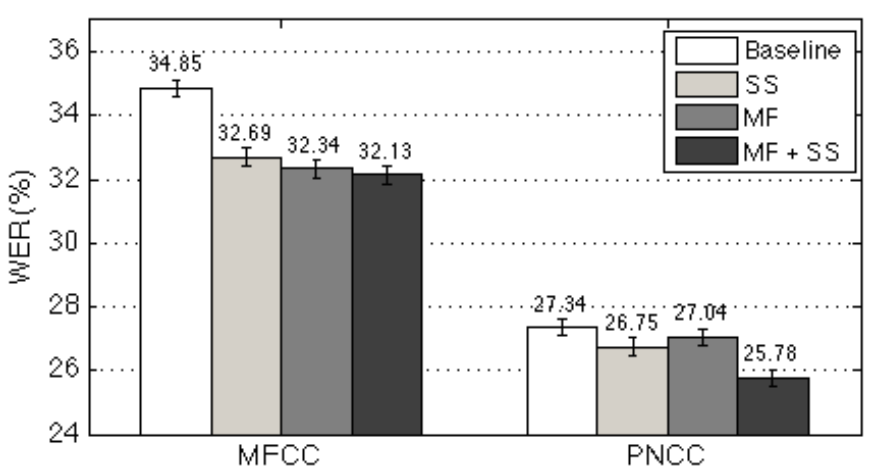

Fig. 10. Recognition results in terms of WER[\%] and 95\% confidence intervals using a noisy contaminated version of the WSJ0 dataset (average over all the noises and SNR).

obtained by the baselines and $\mathrm{MF}+\mathrm{SS}$ are shown in these figures.

Figure 10 shows that: (1) The PNCC spectral representation baseline clearly outperforms the corresponding $M F C C$ baseline; (2) the application of MF improves the baseline recognition rates but not in a significant way for the PNCC case; (3) the joint use of SS and MF improves the recognition rates obtained with $\mathrm{SS}$ and with the baseline in a statistically significant manner for both representations; (4) the PNCC (baseline, SS, MF, SS+MF) achieve in all cases better recognition rates than the corresponding features based on $M F C C$, and (5) the improvements in the WSJ0 dataset are lower than the AURORA and ISOLET datasets. We suggest that this reduction is due to the larger size of the database and the influence of the language models in the acoustic decoding process.

Note also, from Figure 11, that the PNCC (MF+SS) method achieves the best performance in every noise and SNR conditions. The improvement in white noise in Figure 11 (a), and speaker noise in Figure 11 (c) conditions are particularly worth 


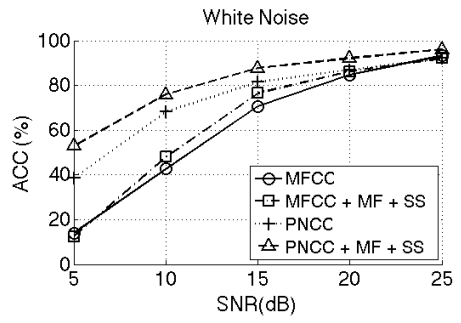

(a)

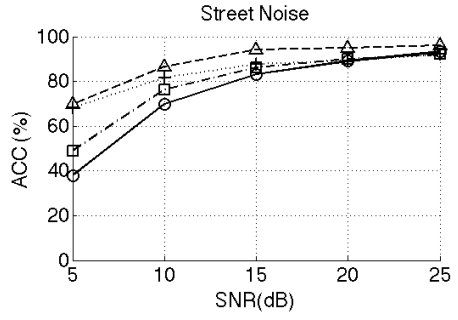

(b)

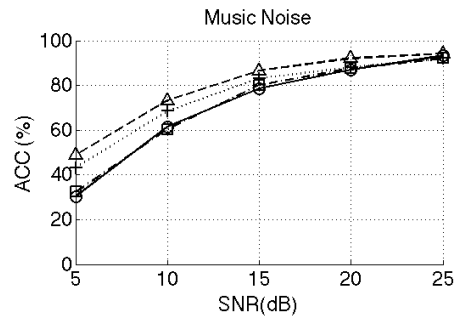

(c)

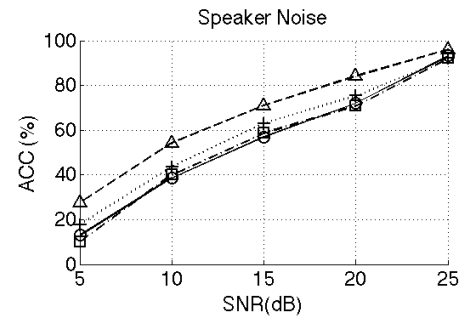

(d)

Fig. 11. Recognition results obtained under different additive noise conditions in terms of ACC[\%] using the WSJ0 dataset

TABLE I

AVERAGE RUNTIME PER UTTERANCE FOR THE DIFFERENT METHODS OVER ALL TESTING SETS ON THE AURORA 2 DATASET.

\begin{tabular}{|l|c|c|}
\hline Method & Time (ms) & \% from baseline \\
\hline MFCC Baseline & 19.66 & - \\
MFCC + SS & 26.98 & $37.23 \%$ \\
MFCC + MF & 21.84 & $11.80 \%$ \\
MFCC + MF + SS & 28.82 & $46.59 \%$ \\
PNCC Baseline & 67.93 & - \\
PNCC+ SS & 85.69 & $26.14 \%$ \\
PNCC + MF & 69.45 & $2.23 \%$ \\
PNCC + MF + SS & 87.06 & $28.16 \%$ \\
\hline
\end{tabular}

noticing, since the proposed method clearly outperforms the PNCC baseline.

\section{E. Computational complexity}

Table I shows a comparison of the runtime for the different methods under different conditions (clean and noisy speech), using a workstation with $3.4 \mathrm{GHz}$ Intel Core i7 and $16 \mathrm{~GB}$ of RAM memory. The running times were obtained by averaging each of the utterances over all testing sets on the AURORA 2 dataset. The extra time added by MF is relatively low for either MFCC or PNCC. It is worth noting that the time spent by MFCC $+\mathrm{MF}+\mathrm{SS}$ is below the PNCC baseline, despite obtaining similar results in almost every noisy condition.

\section{CONCLUSIONS AND FURTHER WORK}

In this paper we present an enhanced, perceptuallymotivated SE for morphological filtering of speech that models the complexity of HAS masking properties. Well-known empirical data in either temporal or frequency domains were interpolated to produce a three-dimensional SE for morphological filtering. A smoothness constraint was imposed since this is more suited for our hypothesis that the morphological closing operation produces a convexification of the spectro-temporal envelope of speech that models the masking properties of the HAS.

Despite ingrained intuitions that this imitation of auditory masking degrades the quality of the extracted features producing a blurring effect, the results we have obtained indicate that it could be in fact a sophisticated mechanism for selecting the most important parts of the spectrum from an intelligibility point of view, taking away irrelevant information and emphasizing the most robust parts of the spectrum.
The application of morphological processing with this SE in conjunction with the Power-Normalized spectro-temporal representation produces a significant increase in recognition rates in Aurora 2, ISOLET and a noisy contaminated version of the Wall Street Journal datasets. Also the results show that our method improves the recognition rates in both hybrid and traditional HMM based back-ends. To reach these results we have tested the combination of PNCC, spectral subtraction and morphological processing.

Future work will focus on the introduction of the dependency of the masker strength into morphological filtering and its interaction with alternative acoustic models such as those based on Deep Neural Networks (DNN).

\section{ACKNOWLEDGEMENTS}

This contribution has been supported by an Airbus Defense and Space Grant (Open Innovation - SAVIER) and Spanish Government-CICYT projects TEC2014-53390-P and TEC2014-61729-EXP. We would also like to thank Chanwoo Kim for kindly providing the testing noises for the WSJO dataset and PNCC source code.

\section{REFERENCES}

[1] S. Davis and P. Mermelstein, "Comparison of parametric representations for monosyllabic word recognition in continuously spoken sentences," Acoustics, Speech and Signal Processing, IEEE Transactions on, vol. 28, no. 4, pp. 357-366, Aug 1980.

[2] H. Fletcher and W. A. Munson, "Loudness, its definition, measurement and calculation," The Journal of the Acoustical Society of America, vol. 5, no. 2, pp. 82-108, October 1933.

[3] H. Hermansky, B. Hanson, and H. Wakita, "Perceptually based linear predictive analysis of speech," in Acoustics, Speech and Signal Processing (ICASSP), 1985 IEEE International Conference on, vol. 10, Apr 1985, pp. 509-512.

[4] D.-S. Kim, S.-Y. Lee, and R. Kil, "Auditory processing of speech signals for robust speech recognition in real-world noisy environments," Speech and Audio Processing, IEEE Transactions on, vol. 7, no. 1, pp. 55-69, Jan 1999.

[5] A. Ali, J. Van der Spiegel, and P. Mueller, "Robust auditory-based speech processing using the average localized synchrony detection," Speech and Audio Processing, IEEE Transactions on, vol. 10, no. 5, pp. 279-292, Jul 2002.

[6] U. H. Yapanel and J. H. Hansen, "A new perceptually motivated mvdr-based acoustic front-end (pmvdr) for robust automatic speech recognition," Speech Communication, vol. 50, no. 2, pp. 142 - 152, 2008. [Online]. Available: http://www.sciencedirect.com/science/article/ pii/S0167639307001434

[7] F. Müller and A. Mertins, "Contextual invariant-integration features for improved speaker-independent speech recognition," Speech Communication, vol. 53, no. 6, pp. $830-841,2011$. 
[8] N. Moritz, J. Anemuller, and B. Kollmeier, "Amplitude modulation spectrogram based features for robust speech recognition in noisy and reverberant environments," in Acoustics, Speech and Signal Processing (ICASSP), 2011 IEEE International Conference on, May 2011, pp. 5492-5495.

[9] A. Fazel and S. Chakrabartty, "Sparse auditory reproducing kernel (spark) features for noise-robust speech recognition," Audio, Speech, and Language Processing, IEEE Transactions on, vol. 20, no. 4, pp. 1362-1371, May 2012.

[10] H. Hermansky and N. Morgan, "Rasta processing of speech," Speech and Audio Processing, IEEE Transactions on, vol. 2, no. 4, pp. 578589, Oct 1994.

[11] S. Seneff, "A joint synchrony/mean-rate model of auditory speech processing," in Readings in Speech Recognition, A. Waibel and K.-F. Lee, Eds. San Francisco, CA, USA: Morgan Kaufmann Publishers Inc., 1990, pp. 101-111. [Online]. Available: http: //dl.acm.org/citation.cfm?id=108235.108241

[12] O. Ghitza, "Auditory nerve representation as a front-end for speech recognition in a noisy environment," Comput. Speech Lang., vol. 1, no. 2, pp. 109-130, Dec. 1986. [Online]. Available: http://dx.doi.org/10.1016/S0885-2308(86)80018-3

[13] J. Allen, "Cochlear modeling," ASSP Magazine, IEEE, vol. 2, no. 1, pp 3-29, Jan 1985 .

[14] R. Lyon, "A computational model of filtering, detection, and compression in the cochlea," in Acoustics, Speech and Signal Processing (ICASSP), 1982 IEEE International Conference on, vol. 7, May 1982, pp. $1282-1285$.

[15] - "A computational model of binaural localization and separation," in Acoustics, Speech and Signal Processing (ICASSP), 1983 IEEE International Conference on, vol. 8, Apr 1983, pp. 1148-1151.

[16] C. Kim and R. Stern, "Power-normalized cepstral coefficients (PNCC) for robust speech recognition," in Acoustics, Speech and Signal Processing (ICASSP), 2012 IEEE International Conference on, March 2012, pp. $4101-4104$.

[17] C. Kim and R. M. Stern, "Power-normalized cepstral coefficients (PNCC) for robust speech recognition," IEEE Transactions on Audio, Speech, and Language Processing.

[18] M. G. Heinz, X. Zhang, I. C. Bruce, and L. H. Carney, "Auditory nerve model for predicting performance limits of normal and impaired listeners," Acoustics Research Letters Online, vol. 2, no. 3, pp. 91-96, 2001.

[19] K. Paliwal and B. T. Lilly, "Auditory masking based acoustic frontend for robust speech recognition," in TENCON '97. IEEE Region 10 Annual Conference. Speech and Image Technologies for Computing and Telecommunications., Proceedings of IEEE, vol. 1, 1997, pp. 165-168 vol.1.

[20] Y. Hu and P. Loizou, "Incorporating a psychoacoustical model in frequency domain speech enhancement," Signal Processing Letters, IEEE, vol. 11 , no. 2, pp. 270-273, 2004.

[21] S. Haque, "Utilizing auditory masking in automatic speech recognition," in Audio Language and Image Processing (ICALIP), 2010 International Conference on, 2010, pp. 1758-1764.

[22] B. T. Meyer and B. Kollmeier, "Robustness of spectro-temporal features against intrinsic and extrinsic variations in automatic speech recognition," Speech Commun., vol. 53, no. 5, pp. 753-767, 2011.

[23] B. T. Meyer, C. Spille, B. Kollmeier, and N. Morgan, "Hooking up spectro-temporal filters with auditory-inspired representations for robust automatic speech recognition," in Proceedings of Interspeech (13th International Conference on Speech Communication and Technology), 2012.

[24] B. T. Meyer, S. V. Ravuri, M. R. Schdler, and N. Morgan, "Comparing different flavors of spectro-temporal features for ASR," in Proceedings of Interspeech (12th International Conference on Speech Communication and Technology), 2011, pp. 1269-1272.

[25] M. Heckmann, X. Domont, F. Joublin, and C. Goerick, "A hierarchical framework for spectro-temporal feature extraction," Speech Communication, vol. 53, no. 5, pp. 736 - 752, 2011.

[26] A. Hurmalainen and T. Virtanen, "Modelling spectro-temporal dynamics in factorisation-based noise-robust automatic speech recognition," in Acoustics, Speech and Signal Processing (ICASSP), 2012 IEEE International Conference on, 2012, pp. 4113-4116.

[27] C. Martínez, J. Goddard, D. Milone, and H. Rufiner, "Bioinspired sparse spectro-temporal representation of speech for robust classification," Computer Speech and Language, vol. 26, no. 5, pp. 336 - 348, 2012.

[28] R. Stern and N. Morgan, "Hearing is believing: Biologically inspired methods for robust automatic speech recognition," Signal Processing Magazine, IEEE, vol. 29, no. 6, pp. 34-43, 2012.
[29] M. Berouti, R. Schwartz, and J. Makhoul, "Enhancement of speech corrupted by acoustic noise," in Acoustics, Speech and Signal Processing (ICASSP), 1979 IEEE International Conference on, vol. 4, 1979, pp. 208-211.

[30] P. Scalart and J. Filho, "Speech enhancement based on a priori signal to noise estimation," in Acoustics, Speech and Signal Processing (ICASSP), 1996 IEEE International Conference on, vol. 2, 1996, pp. 629-632.

[31] Y. Ephraim and D. Malah, "Speech enhancement using a minimummean square error short-time spectral amplitude estimator," Acoustics, Speech and Signal Processing, IEEE Transactions on, vol. 32, no. 6, pp. 1109-1121, 1984

[32] J. Cadore, F. J. Valverde-Albacete, A. Gallardo-Antolín, and C. PeláezMoreno, "Auditory-inspired morphological processing of speech spectrograms: Applications in automatic speech recognition and speech enhancement," Cognitive Computation, vol. 5, no. 4, pp. 426-441, 2013.

[33] J. Cadore, A. Gallardo-Antolín, and C. Peláez-Moreno, "Morphological processing of spectrograms for speech enhancement," in Advances in Nonlinear Speech Processing. Springer-Verlag, 2011, pp. 224-231.

[34] J. Hansen, "Morphological constrained feature enhancement with adaptive cepstral compensation (MCE-ACC) for speech recognition in noise and Lombard effect," Speech and Audio Processing, IEEE Transactions on, vol. 2, no. 4, pp. 598-614, 1994.

[35] H. Fastl and E. Zwicker, Psycho-acoustics: Facts and Models, 3rd ed. Springer, 2007.

[36] F. de-la Calle-Silos, F. J. Valverde-Albacete, A. Gallardo-Antolín, and C. Peláez-Moreno, "ASR feature extraction with morphologicallyfiltered power-normalized cochleograms," in Proceedings of Interspeech (15th International Conference on Speech Communication and Technology), 2014, pp. $2430-2434$.

[37] E. Zwicker and E. Terhardt, "Analytical expressions for critical-band rate and critical bandwidth as a function of frequency," J. Acoust. Soc. Am., vol. 68, p. 1523, 1980.

[38] S. S. Stevens, J. Volkmann, and E. B. Newman, "A scale for the measurement of the psychological magnitude of pitch," J. Acoust. Soc. Am., vol. 8, pp. 185-190, 1937.

[39] B. Glasberg and B. Moore, "Derivation of auditory filter shapes from notched-noise data," Hearing Res., vol. 47, no. 1-2, pp. 103-138, 1990.

[40] B. Moore and B. Glasberg, "A revised model of loudness perception applied to cochlear hearing loss," Hearing Research, vol. 188, no. 1-2, pp. $70-88,2004$.

[41] _ "Suggested formula for calculating auditory-filter bandwidths and excitation patterns," J. Acoust. Soc. Am., vol. 74, p. 750, 1983.

[42] R. Patterson, K. Robinson, J. Holdsworth, D. McKeown, C. Zhang, and M. Allerhand, "Complex sounds and auditory images," Auditory physiology and perception, vol. 83, pp. 429-446, 1992.

[43] R. D. Patterson, K. Robinson, J. Holdsworth, D. Mckeown, C. Zhang, and M. Allerhand, "Complex sounds and auditory images," in in Proc. 9th Int. Symp. Hearing Audit., Physiol. Perception, 1992, pp. 429-446.

[44] G. V. Békésy, "On the resonance curve and the decay period at various points on the cochlear partition," The Journal of the Acoustical Society of America, vol. 21, no. 3, pp. 245-254, 1949.

[45] E. Zwicker and A. Jaroszewski, "Inverse frequency dependence of simultaneous tone-on-tone masking patterns at low levels," The Journal of the Acoustical Society of America, vol. 71, no. 6, pp. 1508-1512, 1982.

[46] W. Jesteadt, S. P. Bacon, and J. Lehman, "Forward masking as a function of frequency, masker level, and signal delay," The Journal of the Acoustical Society of America, vol. 71, no. 4, pp. 950 - 962, 1982.

[47] G. Matheron and J. Serra, "The birth of mathematical morphology," in Proc. 6th Int. Symp. Mathematical Morphology. Sydney, Australia, 2002, pp. 1-16.

[48] E. R. Dougherty and R. A. Lotufo, Hands-on Morphological Image Processing, ser. Tutorial Texts in Optical Engineering. SPIE press, 2003.

[49] J. Cadore, C. Peláez-Moreno, and A. Gallardo-Antolín, "Morphological processing of a dynamic compressive gammachirp filterbank for automatic speech recognition," in IberSPEECH 2012, 2012.

[50] F. de la Calle Silos, "Source code," http://www.tsc.uc3m.es/ fsilos

[51] D. Pearce and H.-G. Hirsch, "The AURORA experimental framework for the performance evaluation of speech recognition systems under noisy conditions," in ICSLP 2000, 6th International Conference on Spoken Language Processing, no. October, 2000, pp. 16-19.

[52] S. Young, G. Evermann, M. Gales, T. Hain, D. Kershaw, X. Liu, G. Moore, J. Odell, D. Ollason, D. Povey, V. Valtchev, and P. Woodland, The HTK book, ver 3.4. Entropic Cambridge Research Laboratory, 2006. 
[53] K. Bache and M. Lichman, "The isolet spoken letter database," last accessed: 2015-07-02. [Online]. Available: https://archive.ics.uci.edu/ $\mathrm{ml} /$ datasets/ISOLET

[54] D. Gelbart, H. W., M. Holmberg, and N. Morgan, "Noisy isolet and isolet testbeds," last accessed: 2015-07-02. [Online]. Available: http://www1.icsi.berkeley.edu/Speech/papers/eurospeech05-onset/isolet/

[55] H. Bourlard and N. Morgan, "Hybrid HMM/ANN systems for speech recognition: Overview and new research directions," Adaptive Process. of Sequences and Data Structures, pp. 389-417, 1998.

[56] D. Johnson, "ICSI quicknet software package," [on-line] http://www.icsi.berkeley.edu/Speech/qn.html, last accessed: 201507-02.

[57] D. B. Paul and J. M. Baker, "The design for the wall street journal-based csr corpus," in Proceedings of the Workshop on Speech and Natural Language, ser. HLT '91. Stroudsburg, PA, USA: Association for Computational Linguistics, 1992, pp. 357-362. [Online]. Available: http://dx.doi.org/10.3115/1075527.1075614

[58] K. Vertanen, "Baseline WSJ acoustic models for HTK and Sphinx: Training recipes and recognition experiments," Technical report). Cambridge, United Kingdom: Cavendish Laboratory, Tech. Rep., 2006.

[59] G. Hirsch, "FaNT - Filtering and Noise Adding Tool," http://dnt.kr.hsnr. de/download.html, 2005. 